\title{
Time spent in prior hospital stay and outcomes for ventilator patients in long-term acute care hospitals
}

\author{
Berna Demiralp ${ }^{1}$, Lane Koenig ${ }^{1 *}$, Jing $\mathrm{Xu}^{1}$, Samuel Soltoff ${ }^{1}$ and John Votto ${ }^{2}$
}

\begin{abstract}
Background: Long-term acute care hospitals (LTACHs) treat mechanical ventilator patients who are difficult to wean and expected to be on mechanical ventilator for a prolonged period. However, there are varying views on who should be transferred to LTACHs and when they should be transferred. The purpose of this study is to assess the relationship between length of stay in a short-term acute care hospital (STACH) after endotracheal intubation (time to LTACH) and weaning success and mortality for ventilated patients discharged to an LTACH.

Methods: Using 2014-2015 Medicare claims and assessment data, we identified patients who had an endotracheal intubation in STACH and transferred to an LTACH with prolonged mechanical ventilation (defined as 96 or more consecutive hours on a ventilator). We controlled for age, gender, STACH stay procedures and diagnoses, Elixhauser comorbid conditions, and LTACH quality characteristics. We used instrumental variable estimation to account for unobserved patient and provider characteristics.
\end{abstract}

Results: The study cohort included 13,622 LTACH cases with median time to LTACH of 18 days. The unadjusted ventilator weaning rate at LTACH was $51.7 \%$, and unadjusted 90 -day mortality rate was $43.7 \%$. An additional day spent in STACH after intubation is associated with $11.6 \%$ reduction in the odds of weaning, representing a 2.5 percentage point reduction in weaning rate at 18 days post endotracheal intubation. We found no statistically significant relationship between time to LTACH and the odds of 90-day mortality.

Conclusions: Discharging ventilated patients earlier from STACH to LTACH is associated with higher weaning probability for LTACH patients on prolonged mechanical ventilation. Our findings suggest that delaying ventilated patients' discharge to LTACH may negatively influence the patients' chances of being weaned from the ventilator.

Keywords: Ventilator weaning, Mortality, Length of stay

\section{Background}

The number of critically ill patients receiving ventilator care has grown considerably over the last several years [1]. While the use of ventilator support has extended the life of patients, those who survive face poor prognosis, with high 1-year mortality rates [2, 3]. A key step

*Correspondence: Lane.koenig@knghealth.com

1 KNG Health Consulting, LLC, 15245 Shady Grove Road, \# 365, Rockville,

MD 20850, USA

Full list of author information is available at the end of the article to maximize the recovery of these patients is liberating them from the ventilator. However, only about half of patients are liberated from mechanical ventilation upon hospital discharge [3].

In the U.S., many critically ill patients on ventilators are discharged to long-term acute care hospitals (LTACHs) to wean off the ventilator. These specialty hospitals, of which there are fewer than 400 in the nation, have an average length of stay of over 25 days and care for critically ill patients requiring an extended inpatient hospital

(c) The Author(s) 2021. Open Access This article is licensed under a Creative Commons Attribution 4.0 International License, which permits use, sharing, adaptation, distribution and reproduction in any medium or format, as long as you give appropriate credit to the original author(s) and the source, provide a link to the Creative Commons licence, and indicate if changes were made. The images or other third party material in this article are included in the article's Creative Commons licence, unless indicated otherwise in a credit line to the material. If material is not included in the article's Creative Commons licence and your intended use is not permitted by statutory regulation or exceeds the permitted use, you will need to obtain permission directly from the copyright holder. To view a copy of this licence, visit http://creativecommons.org/licenses/by/4.0/. The Creative Commons Public Domain Dedication waiver (http://creativeco mmons.org/publicdomain/zero/1.0/) applies to the data made available in this article, unless otherwise stated in a credit line to the data. 
stay [4]. In 2017, roughly $44 \%$ of Medicare fee-for-service (FFS) patients on prolonged mechanical ventilation (PMV) (defined as 96 or more consecutive hours on a ventilator) and discharged alive from a short-term acute care hospital (STACH) to a post-acute care setting were admitted to an LTACH, compared to $3 \%$ of all hospitalized Medicare FFS patients. ${ }^{1}$

To support discharge decisions, many hospitals and payers use proprietary or commercial clinical guidelines to help in patient placement assessments. These guidelines provide criteria for when it is appropriate for a ventilated patient to be admitted to an LTACH. In 2005, a consensus statement from the National Association for Medical Direction of Respiratory Care (NAMDRC) recommended that prolonged mechanical ventilation be defined as the need for 21 consecutive days or longer on mechanical ventilation for at least $6 \mathrm{~h}$ a day (abbreviated "21-day requirement") [5]. Based on this recommendation, private health plans covering Medicare beneficiaries (Medicare Advantage plans) often cite failure to meet the 21-day requirement as a key reason for prior-authorization denials for admission to an LTACH.

In this study, we assess the relationship between length of stay in a STACH after endotracheal intubation and mortality and weaning success for ventilated patients discharged to an LTACH. Earlier discharged to an LTACH for ventilator weaning services may have two conflicting effects. First, patients transferred earlier may experience improved outcomes due to receipt of specialized ventilator weaning services at LTACHs. On the other hand, if early transfer patients are less stable, requiring a level of care only available in an intensive care unit (ICU), discharging ventilated patients too early to an LTACH may negatively affect outcomes. We use an instrumental variable approach to conduct the analysis in recognition that STACH discharge decisions may be influenced by patient complexity and financial consideration not readily observed in administrative data.

\section{Methodology}

We constructed our study cohort, explanatory variables, and outcomes using 2014-2015 Medicare claims and assessment data. The Medicare claims data were used to study services provided by STACHs, LTACHs, inpatient rehabilitation facilities (IRFs), skilled nursing facilities (SNFs), and home health agencies (HHAs). We used assessment data from the Minimum Data Set to analyze stays in nursing homes (NHs). We obtained dates of admissions and discharges, patient demographic information, and clinical diagnosis codes and procedure

${ }^{1}$ Based on authors' analysis of Medicare FFS claims data. codes for each hospitalization and LTACH transfer. We used LTACH Compare data available on the Centers for Medicare \& Medicaid (CMS) website to obtain LTACH characteristics on quality during 2014 and 2015. Ethical \& Independent Review Services determined that a waiver from the requirement to obtain HIPPA authorization was justified for this research project.

Our study population consisted of Medicare beneficiaries who were aged 65 or older, discharged from a STACH between January 1, 2014 and June 30, 2015, and, subsequently, admitted to an LTACH. We limited the analytic sample to Medicare beneficiaries who: (1) received a tracheostomy (ICD 9 procedure codes: "311","312","3121","3129") at the STACH; (2) were on prolonged mechanical ventilation at the STACH and LTACH; (3) spent at least one day in an ICU during the STACH stay; (4) were admitted to an LTACH same or next day after discharge from STACH; and (5) died in the LTACH or were discharged from the LTACH to another hospital, IRF, SNF, NH, hospice, or home with or without home health care.

Our sample exclusions included: (1) beneficiaries who had Medicare Advantage coverage during the month of LTACH stay and the following month; (2) beneficiaries who did not have continuous Part A coverage during the month of LTACH stay and the following month; (3) beneficiaries who were admitted to a STACH for the non-surgical treatment of cancer; (4) beneficiaries who had conditions that were not suitable to wean, such as quadriplegia, or amyotrophic lateral sclerosis; (5) beneficiaries who discharged from a STACH or LTACH against medical advice; (6) beneficiaries who exhausted Medicare benefits during a STACH or LTACH stay; and (7) beneficiaries who had overlapping stays in a STACH or LTACH based on dates of admission and discharge in claims or had a missing admission or discharge date. We also excluded outlier cases in terms of days from intubation at the STACH to LTACH transfer ("time to LTACH"), defined as those with days outside of the 1st and 99th percentiles of the time to LTACH distribution, from the analysis.

We examined two clinical outcomes: ventilator weaning during LTACH stay and mortality. A patient was considered to have weaned off the ventilator if he/she was discharged alive from the LTACH and not on mechanical ventilation in the next observed setting after discharge from the LTACH. We measured mortality within 90 days starting at day of LTACH admission. We performed logistic regression to estimate the outcomes.

The key explanatory variable in the analysis was "time to LTACH" (TTL), which was defined as the length of time between endotracheal intubation (ICD 9 procedure code: "9604") in the STACH to discharge from STACH. 
We adapted a risk-adjustment model developed by Kahn et al. to examine in-LTACH mortality for a ventilated population [6]. In addition to TTL, we obtained clinical information from the STACH stay and constructed risk-adjustment variables based on the model developed by Kahn et al. for 90-day mortality rates among patients admitted to LTACHs for ventilator weaning. Patient characteristics included: age, gender, STACH stay procedures and diagnoses (coronary artery bypass graft (CABG), dialysis, hypotension, percutaneous transluminal coronary angioplasty (PTCA), stroke, thrombocytopenia, trauma, and valve replacement), and Elixhauser comorbid conditions during STACH stay. We also controlled for two CMS LTACH quality measures obtained from LTACH Compare: 30-day all-cause unplanned readmission rate and percent of patients with new or worsened pressure ulcers [7]. We added these quality proxy measures, which are readily available from CMS and validated, to control for differences in patient outcomes that may be related to LTACH quality and correlated with TTL.

TTL may be correlated with factors unobservable from claims and assessment data, such as STACH preferences, STACH quality, payer differences, and patient severity. The direction of the correlation is unclear as factors determining TTL may be complex. For example, patients who stay longer in the acute care hospital may be sicker or it may reflect STACH physician preference to keep the patient under his or her direct care longer. On the other hand, patients who have shorter lengths of stay may be less acutely ill or debilitated or may be discharged sooner by hospitals to limit financial losses on complex patients. We accounted for the potential endogeneity of TTL by using instrumental variable (IV) estimation. The IV used in the analysis was the residual from a linear regression of LTACH daily census of all patients on LTACH daily census of ventilator patients. By constructing the IV based on this regression residual, our IV is not correlated with LTACH daily census of ventilator patients, which may be correlated with outcomes if there is a volume-quality relationship for treating ventilator patients.

Since the models for outcomes were non-linear, we used two-stage residual inclusion (2SRI) estimation to conduct the IV analysis [8]. In the first stage, we used TTL as the dependent variable, and we included IV and all the other covariates as independent variables. The model we used was generalized linear model (GLM) with a Gaussian distribution, a log-link function and robust standard errors. We obtained the residuals from the GLM to include in the second stage. Specifically, in the second stage, we use logistic regression, in which the outcome variables were weaning or 90-day mortality, and the explanatory variables included TTL, residual from the first stage GLM, age categories, gender, and all the clinical conditions and comorbidities. We used bootstrapping (500 replications) to approximate the asymptotically correct standard errors.

For the weaning outcome, we conducted two sets of sensitivity analysis. In the first set of sensitivity analysis, we only considered a patient to have weaned if he/she met the original definition (i.e., patient discharged alive from the LTACH and not on mechanical ventilation in the next setting after LTACH discharge) and the patient was alive in the next setting for at least 7 days. In the second set of sensitivity analysis, we excluded patients who went to hospice after LTACH discharge from our analytic sample.

For the logistic regression and the 2SRI IV analysis, we calculated the odds ratio and marginal effects for TTL. We considered a $p$ value less than 0.05 to be statistically significant. All the analyses were performed with STATA 16.0 (StataCorp LLC, College Station, Texas).

\section{Results}

Our study cohort included 13,622 Medicare beneficiaries who had an endotracheal intubation in a STACH and were discharged to an LTACH between January 1, 2014 and June 30, 2015 (Table 1). Average length of stay in the STACH prior to admission to the LTACH was 24.0 days, and the average number of days between endotracheal intubation and discharge from STACH was 19.6 days. The number of days to LTACH after endotracheal intubation had a median of 18 and interquartile range of $[13,24]$. Some of the common diagnoses in the study cohort included congestive heart failure (45.8\%), cardiac arrhythmias (52.7\%), chronic pulmonary disease (48.3\%), and fluid and electrolyte disorders (78.7\%).

Patients who were weaned off the ventilator at the LTACH, on average, were younger (68.7 vs. 72.7$)$ and had shorter time in STACH between endotracheal intubation and discharge to LTACH (19.1 days vs. 20.2 days). In addition, patients who weaned were less likely to have 19 of the 31 Elixhauser comorbidities examined. Similar to patients who weaned, patients who survived the 90-day period post LTACH admission were younger (68.6 vs. 73.2) with shorter TTL (19.2 vs. 20.2 days). They were also less likely to have 20 of the 31 Elixhauser comorbidities examined.

The unadjusted ventilator weaning rate at LTACHs in our study cohort is $51.7 \%$, indicating that more than half of the patients in our study weaned from the ventilator at the LTACH and were discharged alive (Table 2). Weaning rates were lower in our sensitivity analyses. Unadjusted weaning rate was 47.1 and $48.0 \%$ when weaned patients were required to remain alive for 7 days following discharge from LTACH or when excluding patients discharged from LTACH to hospice, 
Table 1 Patient characteristics

\begin{tabular}{|c|c|c|c|c|c|}
\hline & All & Weaned at LTACH & $\begin{array}{l}\text { Not Weaned at } \\
\text { LTACH }\end{array}$ & Alive in 90 Days & Expired in 90 Days \\
\hline No. of Cases & 13,622 & 7046 & 6576 & 7668 & 5954 \\
\hline \multicolumn{6}{|l|}{ Time to LTACH } \\
\hline Mean & 19.6 & 19.1 & 20.2 & 19.2 & 20.2 \\
\hline 25th percentile & 13.0 & 13.0 & 14.0 & 13.0 & 14.0 \\
\hline Median & 18.0 & 17.0 & 18.0 & 18.0 & 18.0 \\
\hline 75th percentile & 24.0 & 23.0 & 25.0 & 23.0 & 25.0 \\
\hline Age & 70.6 & 68.7 & 72.7 & 68.6 & 73.2 \\
\hline Male & $50.4 \%$ & $50.2 \%$ & $50.7 \%$ & $49.3 \%$ & $51.9 \%$ \\
\hline Prior STACH LOS & 24.0 & 23.2 & 24.9 & 23.3 & 25.0 \\
\hline \multicolumn{6}{|l|}{ Prior STACH Procedures and Diagnoses } \\
\hline Dialysis & $15.2 \%$ & $13.2 \%$ & $17.5 \%$ & $12.4 \%$ & $18.9 \%$ \\
\hline PTCA & $1.6 \%$ & $1.4 \%$ & $1.7 \%$ & $1.6 \%$ & $1.5 \%$ \\
\hline CABG & $3.9 \%$ & $3.8 \%$ & $4.1 \%$ & $3.6 \%$ & $4.4 \%$ \\
\hline Valve Replacement & $2.8 \%$ & $2.6 \%$ & $3.0 \%$ & $2.4 \%$ & $3.3 \%$ \\
\hline Hypotension & $10.9 \%$ & $11.2 \%$ & $10.6 \%$ & $11.1 \%$ & $10.6 \%$ \\
\hline Thrombocytopenia & $15.9 \%$ & $15.5 \%$ & $16.3 \%$ & $15.0 \%$ & $17.0 \%$ \\
\hline Stroke & $13.5 \%$ & $15.0 \%$ & $12.0 \%$ & $14.1 \%$ & $12.7 \%$ \\
\hline Trauma & $9.8 \%$ & $10.7 \%$ & $8.8 \%$ & $10.2 \%$ & $9.3 \%$ \\
\hline \multicolumn{6}{|l|}{ Exlihauser Comorbidity } \\
\hline Congestive Heart Failure & $45.8 \%$ & $40.5 \%$ & $51.4 \%$ & $41.6 \%$ & $51.1 \%$ \\
\hline Cardiac Arrhythmias & $52.7 \%$ & $48.6 \%$ & $57.0 \%$ & $49.3 \%$ & $57.0 \%$ \\
\hline Valvular Disease & $12.3 \%$ & $10.6 \%$ & $14.2 \%$ & $10.7 \%$ & $14.4 \%$ \\
\hline Pulmonary Circulation Disorders & $15.4 \%$ & $13.6 \%$ & $17.2 \%$ & $14.6 \%$ & $16.4 \%$ \\
\hline Peripheral Vascular Disorders & $12.1 \%$ & $11.5 \%$ & $12.7 \%$ & $11.2 \%$ & $13.3 \%$ \\
\hline Hypertension, Uncomplicated & $39.3 \%$ & $42.7 \%$ & $35.7 \%$ & $43.0 \%$ & $34.7 \%$ \\
\hline Paralysis & $8.9 \%$ & $10.3 \%$ & $7.5 \%$ & $10.1 \%$ & $7.4 \%$ \\
\hline Other Neurological Disorders & $44.3 \%$ & $44.6 \%$ & $44.1 \%$ & $44.7 \%$ & $43.8 \%$ \\
\hline Chronic Pulmonary Disease & $48.3 \%$ & $45.2 \%$ & $51.5 \%$ & $47.3 \%$ & $49.5 \%$ \\
\hline Diabetes, Uncomplicated & $30.0 \%$ & $30.3 \%$ & $29.7 \%$ & $29.7 \%$ & $30.3 \%$ \\
\hline Diabetes, Complicated & $8.9 \%$ & $8.3 \%$ & $9.6 \%$ & $8.2 \%$ & $9.8 \%$ \\
\hline Hypothyroidism & $13.9 \%$ & $13.8 \%$ & $14.0 \%$ & $13.3 \%$ & $14.5 \%$ \\
\hline Renal Failure & $30.2 \%$ & $26.3 \%$ & $34.3 \%$ & $25.9 \%$ & $35.7 \%$ \\
\hline Liver Disease & $9.1 \%$ & $9.0 \%$ & $9.3 \%$ & $8.7 \%$ & $9.7 \%$ \\
\hline Peptic Ulcer Disease Excluding Bleeding & $1.7 \%$ & $1.7 \%$ & $1.7 \%$ & $1.8 \%$ & $1.6 \%$ \\
\hline AIDS/HIV & $0.4 \%$ & $0.4 \%$ & $0.5 \%$ & $0.4 \%$ & $0.5 \%$ \\
\hline Lymphoma & $1.1 \%$ & $1.0 \%$ & $1.2 \%$ & $0.8 \%$ & $1.4 \%$ \\
\hline Metastatic Cancer & $1.4 \%$ & $0.9 \%$ & $2.1 \%$ & $0.8 \%$ & $2.3 \%$ \\
\hline Solid Tumor Without Metastasis & $3.7 \%$ & $2.6 \%$ & $4.9 \%$ & $2.4 \%$ & $5.4 \%$ \\
\hline Rheumatoid Arthritis/Collagen Vascular & $3.7 \%$ & $3.9 \%$ & $3.4 \%$ & $3.8 \%$ & $3.5 \%$ \\
\hline Coagulopathy & $19.9 \%$ & $19.3 \%$ & $20.5 \%$ & $18.6 \%$ & $21.5 \%$ \\
\hline Obesity & $21.3 \%$ & $23.4 \%$ & $19.0 \%$ & $24.0 \%$ & $17.7 \%$ \\
\hline Weight Loss & $42.8 \%$ & $40.9 \%$ & $44.8 \%$ & $40.5 \%$ & $45.7 \%$ \\
\hline Fluid and Electrolyte Disorders & $78.7 \%$ & $78.6 \%$ & $78.9 \%$ & $78.4 \%$ & $79.2 \%$ \\
\hline Blood Loss Anemia & $1.8 \%$ & $1.8 \%$ & $1.7 \%$ & $1.7 \%$ & $1.8 \%$ \\
\hline Deficiency Anemia & $4.1 \%$ & $4.5 \%$ & $3.7 \%$ & $4.4 \%$ & $3.7 \%$ \\
\hline Alcohol Abuse & $5.8 \%$ & $6.9 \%$ & $4.6 \%$ & $6.7 \%$ & $4.7 \%$ \\
\hline Drug Abuse & $3.2 \%$ & $4.3 \%$ & $2.0 \%$ & $4.1 \%$ & $2.1 \%$ \\
\hline Psychoses & $3.1 \%$ & $3.8 \%$ & $2.4 \%$ & $3.6 \%$ & $2.5 \%$ \\
\hline Depression & $11.6 \%$ & $13.1 \%$ & $10.0 \%$ & $13.3 \%$ & $9.5 \%$ \\
\hline Hypertension, Complicated & $28.4 \%$ & $25.0 \%$ & $32.1 \%$ & $24.4 \%$ & $33.5 \%$ \\
\hline Number of Elixhauser comorbidities (mean) & 6.0 & 5.9 & 6.2 & 5.9 & 6.2 \\
\hline
\end{tabular}


Table 1 (continued)

All of the variables in Table 1, except for the prior STACH LOS and the number of Elixhauser comorbidities, were included in the risk adjustment model. Age categories were included as indicator variables (age categories: $<=70,71-75,76-80,81+$ )

Table 2 Unadjusted outcomes

\begin{tabular}{|c|c|c|c|}
\hline & Overall sample size & Outcome rate & $\begin{array}{l}\text { Correlation coefficient } \\
\text { between outcome and time } \\
\text { to LTACH }\end{array}$ \\
\hline Weaned at LTACH & 13,622 & 0.517 & -0.0629 \\
\hline $\begin{array}{l}\text { Weaned at LTACH and alive for at least } \\
7 \text { days after discharge }\end{array}$ & 13,622 & 0.471 & -0.0623 \\
\hline $\begin{array}{l}\text { Weaned at LTACH, excluding patients } \\
\text { discharged to hospice }\end{array}$ & 13,202 & 0.480 & -0.0652 \\
\hline 90-day Mortality & 13,622 & 0.437 & 0.0570 \\
\hline
\end{tabular}

Table 3 Relationship between Time to LTACH and Outcomes

\begin{tabular}{|c|c|c|c|c|c|c|}
\hline \multirow[t]{2}{*}{ Outcomes } & \multicolumn{2}{|c|}{ Univariate Logistic Regression } & \multicolumn{2}{|c|}{ Multivariate Logistic Regression } & \multicolumn{2}{|c|}{$\begin{array}{l}\text { Instrumental Variable } \\
\text { Regression }\end{array}$} \\
\hline & Odds ratio & $P$ value & Odds ratio & $P$ value & Odds ratio & $P$ value \\
\hline Weaned at LTACH & 0.986 & $<0.001$ & 0.990 & $<0.001$ & 0.884 & 0.005 \\
\hline $\begin{array}{l}\text { Weaned at LTACH and alive for } \\
\text { at least } 7 \text { days after discharge }\end{array}$ & 0.986 & $<0.001$ & 0.990 & $<0.001$ & 0.857 & 0.001 \\
\hline $\begin{array}{l}\text { Weaned at LTACH, exclud- } \\
\text { ing patients discharged to } \\
\text { hospice }\end{array}$ & 0.986 & $<0.001$ & 0.990 & $<0.001$ & 0.866 & 0.001 \\
\hline 90-day Mortality & 1.013 & $<0.001$ & 1.007 & 0.001 & 1.046 & 0.327 \\
\hline
\end{tabular}

respectively. The 90 -day mortality rate was 43.7 percent. The correlation coefficient between TTL and ventilator weaning ranged between -0.0623 and -0.0652 (depending on weaning definition), and the correlation coefficient between TTL and 90-day mortality was 0.0570 .

Next, we examined the relationship between TTL and outcomes (probability of weaning and mortality) using logistic regression. Without controlling for any patient characteristics, each additional day in STACH after endotracheal intubation is associated with a $1.4 \%$ decrease in the odds of weaning from the ventilator in the LTACH (Table 3). When we control for patient clinical characteristics, each additional day in STACH after endotracheal intubation is associated with a $1 \%$ reduction in the odds of weaning off the ventilator at the LTACH. This decrease from 1.4 to $1.0 \%$ suggests that patient clinical characteristics accounts for some of the negative relationship between time to LTACH and weaning probability. This relationship holds when weaned patients are limited to those who not only wean from the ventilator but also remain alive for at least 7 days after discharge from LTACH and when the study cohort excludes patients who were discharged from LTACH to hospice.

Our estimation of ventilator weaning probability using IV yielded that an additional day spent in STACH after endotracheal intubation is associated with $11.6 \%$ reduction in the odds of weaning off the ventilator in LTACH (Table 3). If we define ventilator weaning as weaning off of ventilator and being alive for at least 7 days post discharge from LTACH, the odds of weaning decreases by $14.3 \%$ for each additional day in STACH after endotracheal intubation. When we exclude patients discharged from LTACH to hospice, we find that an additional day in STACH is associated with a $13.4 \%$ reduction in odds of weaning off ventilator at LTACH.

Because the models are non-linear, the effects of TTL on outcomes vary at different points in the TTL distribution. To better understand the relationship between TTL and the probability of weaning from ventilator at LTACH, we examined the average predicted probability of weaning if patients were discharged to an LTACH at 13,18 , and 24 days after endotracheal intubation, which correspond to the $25^{\text {th }}, 50^{\text {th }}$, and $75^{\text {th }}$ percentile of the TTL distribution in the study cohort (Table 4). Based 
Table 4 Average Adjusted Outcomes at Select Values of Time to LTACH

\begin{tabular}{|c|c|c|c|c|c|c|}
\hline \multirow[b]{2}{*}{ Time to LTACH } & \multicolumn{2}{|c|}{$\begin{array}{l}\text { Adjusted probability of being weaned } \\
\text { at LTACH }\end{array}$} & \multicolumn{2}{|c|}{$\begin{array}{l}\text { Adjusted probability of being weaned } \\
\text { at LTACH and alive for at least } 7 \text { days } \\
\text { after discharge from LTACH }\end{array}$} & \multicolumn{2}{|c|}{$\begin{array}{l}\text { Adjusted probability of being } \\
\text { weaned at LTACH, excluding } \\
\text { patients discharged to hospice }\end{array}$} \\
\hline & $\begin{array}{l}\text { Logistic Regression } \\
\text { without IV }\end{array}$ & IV Estimation & $\begin{array}{l}\text { Logistic Regression } \\
\text { without IV }\end{array}$ & IV Estimation & $\begin{array}{l}\text { Logistic Regression } \\
\text { without IV }\end{array}$ & IV Estimation \\
\hline 13 days (25th percentile) & 0.534 & 0.667 & 0.486 & 0.651 & 0.496 & 0.651 \\
\hline 17 days & 0.524 & 0.570 & 0.477 & 0.537 & 0.486 & 0.542 \\
\hline 18 days (median) & 0.521 & 0.545 & 0.475 & 0.507 & 0.484 & 0.514 \\
\hline 19 days & 0.519 & 0.520 & 0.473 & 0.478 & 0.481 & 0.487 \\
\hline 24 days (75th percentile) & 0.507 & 0.397 & 0.461 & 0.341 & 0.469 & 0.354 \\
\hline
\end{tabular}

on instrumental variable estimation results, the average predicted probability of weaning is $54.5 \%$ at the median time to LTACH of 18 days post endotracheal intubation. The average adjusted probability of weaning decreases by 2.5 percentage points to $52.0 \%$ at 19 days. The average adjusted probability of being weaned decreases by 27 percentage points from 66.7 to $39.7 \%$ when time to LTACH increases from 13 to 24 days. We consistently observed reductions in the average adjusted probability of weaning with an increase in time to LTACH under alternative definitions of weaning. For example, when weaned patients are defined as those who wean from the ventilator at LTACH and remain alive for at least 7 days post discharge from $\mathrm{LTACH}$, the probability of weaning decreases by 31 percentage points from 65.1 to $34.1 \%$ between 13 and 24 TTL days. When the study cohort excludes patients discharged to hospice, the average predicted probability of weaning decreases by 29.7 percentage points from 65.1 to $35.4 \%$ between 13 and 24 TTL days.

Without controlling for patient characteristics, we found that an additional day in a STACH before discharge to an LTACH is associated with $1.3 \%$ increase in the odds of 90-day mortality among LTACH patients on PMV (OR $=1.013, p$ value $<0.001$ ) (Table 3$)$. Controlling for patient clinical characteristics, an additional TTL day is associated with $0.7 \%$ increase in the odds of 90 -day mortality $(\mathrm{OR}=1.007, p$ value $=0.001)$. When we used instrumental variable estimation, we found no statistically significant relationship between TTL and the odds of 90-day mortality after admission to LTACH $(\mathrm{OR}=1.046, p$ value $=0.327)$.

We conducted diagnostic tests on the instrumental variable to assess its validity. We found that the F-statistic on the excluded instrument in the first-stage regression was 23.34 (25.43 when patients discharged to hospice are excluded) (See Additional file 1: Table S1). This F-statistic is greater than the Stock-Yogo critical value of 16.38, supporting that the instrument is strongly related to TTL. There is no test for the exogeneity of the instrument when there is only one instrumental variable for the one endogenous variable. Although we cannot assess the relationship between the instrument and unobservable characteristics, we examined the relationship between instrument and observable characteristics. Specifically, we divided the TTL into quartiles and assessed whether the average patient characteristics varied across the quartiles. We provide this information in Supplemental Material (Additional file 1: Table S2).

\section{Discussion}

The number of patients on prolonged mechanical ventilation has increased over the recent decades due to advances in intensive care medicine. For example, in the US, rate of endotracheal intubation in the adult population more than doubled between 1993 and 2012 [1]. In the U.S., LTACHs are a common care setting for patients to attempt to wean, after an extended period on a ventilator. However, our understanding of the outcomes for these chronically critically ill patients and how to improve their outcomes has lagged, resulting in an important knowledge gap [3, 9].

In this study, we examine ventilator weaning and mortality rates among Medicare beneficiaries treated with PMV in LTACHs after receiving an endotracheal intubation in a STACH. Our findings contribute to our understanding of both the outcomes for patients with PMV and the determinants of their outcomes. We found that about $51.7 \%$ of patients in our study population weaned from the ventilator at $\mathrm{LTACH} ; 47.1 \%$ of the patients weaned and remained alive for at least 7 days after discharge from LTACH. We also found that more than half $(56.3 \%)$ of the study population was alive within 90-days of being admitted to the LTACH. The median time between endotracheal intubation and admission to LTACH was 18 days, with an interquartile range of 11 days (13 to 24). Our study revealed that the variation in time spent in STACH after endotracheal intubation is associated with the patient's probability of being weaned during the 
subsequent LTACH stay. Patients discharged to LTACHs earlier have higher risk-adjusted odds of weaning. In particular, we found that an additional day spent in STACH after endotracheal intubation reduces the odds of weaning by $11.6 \%$ for patients discharged to LTACHs. This represents a reduction of weaning probability from 54.5 to $52.0 \%$ for patients at median TTL of 18 days. We found no statistically significant relationship between TTL and 90-day mortality.

Our findings are consistent with previous research documenting that patients on PMV have poor prognosis. In their meta-analysis of prior research findings, Damuth and co-authors reported pooled one-year mortality rate of $73 \%$ and ventilator weaning rate of $47 \%$ based on studies that examine outcomes for ventilator patients treated in post-acute hospitals [3]. Compared to previous studies of LTACH patients included in Damuth et al.s metaanalysis, our study has the largest sample size $(13,622$ cases) as it includes Medicare FFS beneficiaries patients treated in all LTACHs in the US during the study period. The largest previous study of LTACH patients by Scheinhorn et al., examining ventilator weaning, included 1,419 patients treated in $23 \mathrm{LTACHs}$ and reported a ventilator weaning rate of $54.1 \%$ [10]. The higher weaning rate in Scheinhorn et al. study as compared to $51.7 \%$ in our study may be partially due to differences in population exclusions between the two studies. Scheinhorn et al. excluded patients admitted to LTACH for end-of-life care (terminal weaning) and for home ventilator training whereas our study did not implement these exclusions.

Our study has limitations that should be considered in reviewing our results. First, due to data limitation, we identify ventilator weaning based on Medicare claims and assessment data in the next care setting following the LTACH stay instead of assessment data from the LTACH. Although the ventilator weaning rate in our study falls within benchmarks from prior studies, including the Ventilation Outcomes Study, the measurement of ventilator weaning based on next setting may have limited accuracy in measuring weaning at LTACH. Second, although we present supporting evidence on the exogeneity of our instrumental variable, we are unable to confirm its exogeneity due to lack of such a test in the case of a single instrument. Third, patients discharged home from LTACH are included in the weaned group although we do not have data on these patients' ventilator use after discharge from LTACH. Since some of the patients discharged home may be on hospice or end-of-life care, we used alternative measures of weaning outcomes that include patients who die soon after LTACH discharge in the non-weaned group. Finally, our analysis is based on patients who are enrolled in traditional Medicare. Therefore, our findings may not be generalizable to patients covered by Medicare Advantage or other commercial plans.

LTACHs are an important care setting for ventilated patients. In 2013, half of all LTACHs had at least $21.7 \%$ of their patients on a ventilator [6]. The introduction of patient criteria in Medicare LTACH payment policy starting in 2016, which reduced payments for cases that did not spend at least 3 days in an intensive care unit prior to admission, has increased the proportion of LTACH patients on a ventilator, further underscoring the importance of LTACHs as specialized treatment facilities for ventilated patients [11]. The number of patients on PMV is likely to continue to grow in the coming years, as a result of continued treatment improvements that keep critical ill patients alive longer and the aging of the U.S. population.

By providing specialized ventilator weaning units, LTACHs may offer benefits to patients and, more broadly, the healthcare system. Shorter TTL may improve weaning outcomes in LTACHs due to: (1) use and earlier implementation of weaning protocols; (2) use and earlier implementation of a rehabilitation model of care, including early and frequent ambulation; and (3) use of multidisciplinary team approach of care which brings together nurses, pharmacists, physicians, nutritionists, pastoral care, social workers and often psychologists. Rak et al. found that high-performing LTACHs in the treatment of ventilated patients differed from lower performers in the promotion of interdisciplinary communication and coordination through, for example, the use of care protocols and interdisciplinary team [12]. Short-term acute care hospitals may be less able to focus on the specific needs of these patients in their intensive care units [13]. In addition, studies have found that hospital volume of ventilated patients may be positively correlated with survival [14-17]. LTACHs may also relieve pressure on hospital intensive care units, reducing over-crowding which may improve outcomes [18].

Even if some ventilated patients may benefit from LTACH care, not all patients on a ventilator in a shortterm acute care hospital may be appropriate to receive care in an LTACH. Some patients may be able to wean off a ventilator at the STACH, while others may have such poor prognosis that weaning is highly unlikely. In these cases, transfer to an LTACH may increase healthcare spending with little measurable benefits. As a result, who should be transferred to an LTACH for ventilator weaning care and the timing of the transfer are key clinical questions in the treatment of patients with PMV requiring investigation. 


\section{Conclusions}

Our findings have important implications for the treatment of chronically critically ill patients on prolonged mechanical ventilation. While LTACHs may offer specialized services and support to treat mechanical ventilator patients who are difficult to wean and expected to be on mechanical ventilator for a prolonged period of time, identifying such patients prospectively based on clinical characteristics has proven to be difficult due to lack of clinical evidence. Partially due to this difficulty in identifying candidates for LTACH care, clinical guidelines have emphasized a "test of time", such as minimum 21 days in a STACH, before transfer to an LTACH. Our findings show that timing of patients' discharge to LTACH may negatively influence the patients' chances of weaning from the ventilator. Earlier access to LTACH care is associated with higher weaning probability for LTACH patients, suggesting patients may benefit from earlier discharge to LTACH and raising questions as to the clinical value of "test of time" approaches to LTACH transfer.

The current COVID-19 pandemic and the widespread use of mechanical ventilation in the treatment of COVID-19 patients has put a spotlight on the outcomes and treatment alternatives of patients on prolonged mechanical ventilation. In preparation for the surge of COVID-19 patients, some have argued that LTACHs can play an important role in freeing up capacity in STACHs by caring for non-COVID-19 patients and serving as overflow setting for COVID-19 patients [19]. Our findings suggest that discharging non-COVID-19 PMV patients earlier to LTACHs during a potential surge of COVID-19 patients may not bring about tradeoffs in patient outcomes. On the contrary, earlier discharge to LTACHs may increase the odds of ventilator weaning for these patients. In addition, while available data are limited, studies indicate that some patients with critical illness from COVID-19 will be intubated and on prolonged ventilation. Some of these patients may face challenges in weaning from the ventilator, and LTACHs are likely to treat some of these patients. An important question for future research is to better understand the appropriate care and care setting for COVID-19 patients on PMV.

\footnotetext{
Abbreviations

FFS: Fee-for-service; GLM: Generalized linear model; HHA: Home health agency; ICU: Intensive care unit; IRF: Inpatient rehabilitation facility; IV: Instrumental variable; LTACH: Long-term acute care hospital; LOS: Length of stay; NAMDRC: National Association for Medical Direction of Respiratory Care; NH: Nursing home; PMV: Prolonged-mechanical ventilation; SNF: Skilled nursing facility; STACH: Short-term acute care hospital; TTL: Time between endotracheal intubation in the STACH and discharge from STACH to LTACH.; 2SRI: Two-stage residual inclusion.
}

\section{Supplementary Information}

The online version contains supplementary material available at https://doi. org/10.1186/s12890-021-01454-1.

Additional file 1. Instrumental Variable Diagnostics.

\section{Authors' contributions}

$\mathrm{BD}, \mathrm{LK}$, and SS designed the study and acquired the data; BD, LK, JX, and SS analyzed the data; $B D, L K, J X$, JV interpreted the findings; $B D, L K$, JX drafted the manuscript, BD, LK, JX, JV revised the manuscript. All authors read and approved the final manuscript.

\section{Funding}

Funding for the study was provided by National Association of Long-Term Hospitals (NALTH). NALTH Research and Quality Committee members reviewed the manuscript and provided feedback. The final decision to incorporate feedback was made by the authors.

\section{Availability of data and materials}

The data used in this study was obtained from Centers for Medicare \& Medicaid Services (CMS) under a Data Use Agreement (DUA), and therefore, they are not publicly available but may be requested by submitting a DUA request to CMS.

\section{Declarations}

\section{Ethics approval and consent to participate}

This research was conducted in accordance with the Declaration of Helsinki. E\&l Review Services, an independent ethical review board, reviewed the study design and determined that formal IRB review is not required (E\&I ID: 17140). E\&l determined that this research fulfilled requirements for a waiver from the requirement to obtain Health Insurance Portability and Accountability Act (HIPAA) Authorization under 45 CFR 164.512(i)(2). Therefore, no further permissions or consent were required to analyze the datasets used in this study.

\section{Consent for publication}

Not applicable.

\section{Competing interests}

BD and LK are employed by KNG Health Consulting, LLC, which has conducted consulting for National Association of Long-Term Hospitals (NALTH). JX, and SS were employed by KNG Health Consulting, LLC at the time the study was conducted. LK serves Director of Policy and Research for NALTH. JV served as the Chief Medical Officer for NALTH.

\section{Author details}

${ }^{1}$ KNG Health Consulting, LLC, 15245 Shady Grove Road, \# 365, Rockville, MD 20850, USA. ${ }^{2}$ Hospital for Special Care, 2150 Corbin Ave, New Britain, CT 06053, USA.

Received: 15 October 2020 Accepted: 2 March 2021

Published online: 24 March 2021

\section{References}

1. Mehta AB, Syeda SN, Wiener RS, Walkey AJ. Epidemiological trends in invasive mechanical ventilation in the United States: a population-based study. J Crit Care. 2015;30(6):1217-21. https://doi.org/10.1016/j.jcrc.2015. 07.007 .

2. Unroe M, Kahn JM, Carson SS, et al. One-year trajectories of care and resource utilization for recipients of prolonged mechanical ventilation: a cohort study. Ann Intern Med. 2010;153(3):167-75. https://doi.org/10. 7326/0003-4819-153-3-201008030-00007.

3. Damuth E, Mitchell JA, Bartock JL, Roberts BW, Trzeciak S. Long-term survival of critically ill patients treated with prolonged mechanical ventilation: a systematic review and meta-analysis. Lancet Respir Med. 2015;3(7):544-53. https://doi.org/10.1016/\$2213-2600(15)00150-2. 
4. Medicare Payment Advisory Commission. Report to the Congress: Medicare Payment Policy. Chapter 11. Long-term care hospital services. March 2020. Accessed on May 1, 2020 http://medpac.gov/docs/default-source/ reports/mar20_entirereport_sec.pdf?sfvrsn $=0$

5. Maclntyre NR, Epstein SK, Carson S, et al. Management of patients requiring prolonged mechanical ventilation: report of a NAMDRC consensus conference. Chest. 2005;128(6):3937-54. https://doi.org/10.1378/chest. 128.6.3937.

6. Kahn JM, Davis BS, Le TQ, Yabes JG, Chang CH, Angus DC. Variation in mortality rates after admission to long-term acute care hospitals for ventilator weaning. J Crit Care. 2018;46:6-12. https://doi.org/10.1016/j. jcrc.2018.03.022.

7. Centers for Medicare \& Medicaid Services. Long-Term Care Hospital Compare. https://www.medicare.gov/longtermcarehospitalcompare/

8. Terza JV. Two-Stage Residual Inclusion Estimation in Health Services Research and Health Economics. Health Serv Res. 2018;53(3):1890-9. https://doi.org/10.1111/1475-6773.12714.

9. Kahn JM. Improving outcomes in prolonged mechanical ventilation: a road map. Lancet Respir Med. 2015;3(7):501-2. https://doi.org/10.1016/ S2213-2600(15)00205-2.

10. Scheinhorn DJ, Hassenpflug MS, Votto JJ, et al. Post-ICU mechanical ventilation at 23 long-term care hospitals: a multicenter outcomes study. Chest. 2007;131(1):85-93.

11. Medicare Payment Advisory Commission. Report to the Congress: Medicare and the Health Care Delivery System. Washington, D.C., June 2019: MedPAC.

12. Rak KJ, Ashcraft LE, Kuza CC, Fleck JC, DePaoli LC, Angus DC, Barnato AE, Castle NG, Hershey TB, Kahn JM. Effective care practices in patients receiving prolonged mechanical ventilation: an ethnographic study. Am J Respir Crit Care Med. 2020;201(7):823-31. https://doi.org/10.1164/rccm. 201910-20060C.

13. Boles JM, Bion J, Connors A, et al. Weaning from mechanical ventilation. Eur Respir J. 2007;29(5):1033-56. https://doi.org/10.1183/09031936.00010 206.
14. Needham DM, Bronskill SE, Rothwell DM, et al. Hospital volume and mortality for mechanical ventilation of medical and surgical patients: a population-based analysis using administrative data. Crit Care Med. 2006;34(9):2349-54. https://doi.org/10.1097/01.CCM.0000233858.85802. $5 \mathrm{C}$.

15. Kahn JM, Goss CH, Heagerty PJ, Kramer AA, O'Brien CR, Rubenfeld GD. Hospital volume and the outcomes of mechanical ventilation. N Engl J Med. 2006;355(1):41-50. https://doi.org/10.1056/NEJMsa053993.

16. Kahn JM, Ten Have TR, Iwashyna TJ. The relationship between hospital volume and mortality in mechanical ventilation: an instrumental variable analysis. Health Serv Res. 2009;44(3):862-79. https://doi.org/10.1111/j. 1475-6773.2009.00959.x.

17. Shahin J, Harrison DA, Rowan KM. Is the volume of mechanically ventilated admissions to UK critical care units associated with improved outcomes? Intensive Care Med. 2014;40(3):353-60. https://doi.org/10. 1007/s00134-013-3205-4.

18. Chen HY, Vanness DJ, Golestanian E. A simplified score for transfer of patients requiring mechanical ventilation to a long-term care hospital. Am J Crit Care. 2011;20(6):e122-30. https://doi.org/10.4037/ajcc2011775 (PMID: 22045148)

19. Makam AN, Grabowski DC. How can we ramp up hospital capacity to handle the surge of covid-19 patients? long-term acute care hospitals can play a critical role. Health Affairs Blog. 2020. https://doi.org/10.1377/ hblog20200410.606195.

\section{Publisher's Note}

Springer Nature remains neutral with regard to jurisdictional claims in published maps and institutional affiliations.
Ready to submit your research? Choose BMC and benefit from:

- fast, convenient online submission

- thorough peer review by experienced researchers in your field

- rapid publication on acceptance

- support for research data, including large and complex data types

- gold Open Access which fosters wider collaboration and increased citations

- maximum visibility for your research: over $100 \mathrm{M}$ website views per year

At BMC, research is always in progress.

Learn more biomedcentral.com/submissions 